\title{
Understanding urinary toxicity after radiotherapy for prostate cancer: first steps forward
}

\author{
Tiziana Rancati ${ }^{1}$, Federica Palorini ${ }^{1}$, Cesare Cozzarini ${ }^{2}$, Claudio Fiorino ${ }^{3}$, Riccardo Valdagni ${ }^{1,4,5}$ \\ ${ }^{1}$ Prostate Cancer Program, Fondazione IRCCS Istituto Nazionale dei Tumori, Milan - Italy \\ ${ }^{2}$ Department of Radiation Oncology, San Raffaele Scientific Institute, Milan - Italy \\ ${ }^{3}$ Department of Medical Physics, San Raffaele Scientific Institute, Milan - Italy \\ ${ }^{4}$ Department of Radiation Oncology 1, Fondazione IRCCS Istituto Nazionale dei Tumori, Milan - Italy \\ ${ }^{5}$ Department of Oncology and Hemato-oncology, Università degli Studi di Milano, Milan, Italy
}

\begin{abstract}
One of the most relevant achievements of Professor Gianni Bonadonna was the implementation of the methodology of controlled clinical trials in medical oncology. It is valid for all cancer types, oncological disciplines and clinical endpoints, both survival and toxicity. This narrative review reports on the status of the current knowledge of the radiation-induced urinary syndrome after external-beam radiotherapy for prostate cancer. In recent years, the syndrome has been the object of large-scale prospective observational trials specifically devoted to investigating the association of patient and treatment features with acute/late urinary toxicity. The first results of these trials allow initial attempts at predictive modeling, which can serve as a basis for the optimization of patient selection and treatment planning.
\end{abstract}

Keywords: Prostate cancer, Radiotherapy, Urinary toxicity

\section{Introduction}

External-beam radiation therapy is one of the leading options in the curative treatment of prostate cancer, either alone or combined with surgery and hormone therapy. In the past decade, many advances have been made in terms of treatment outcomes and reduction of side effects experienced by prostate cancer survivors. Primarily, the introduction of modern linear accelerators allowed the delivery of highly conformal doses to the tumor target through intensitymodulated beams (IMRT), volumetric arcs (volumetric modulated arc therapy, VMAT) and precise image guidance, while reducing the dose to healthy tissues. In addition, deeper knowledge of tissue response to radiation was acquired, especially for rectal and intestinal symptoms, mainly thanks to the establishment of large clinical trials including hundreds of patients, with systematic scoring and follow-up of patient status. It is now well established that different symptoms, even if representing the expression of damage to the same tissue,

\section{Accepted: August 21, 2017}

Published online: September 7, 2017

\section{Corresponding author:}

Tiziana Rancati

Prostate Cancer Program

Fondazione IRCCS Istituto Nazionale dei Tumori

Via Venezian 1

20133 Milan, Italy

tiziana.rancati@istitutotumori.mi.it may be differently related to the absorbed dose. For example, rectal bleeding arises as a serial effect and is mainly related to the absorption of high doses in small rectal mucosa volumes, whilst fecal incontinence is related to intermediate doses absorbed by large rectal volumes.

The development of reliable models of radiation-induced toxicities along with the available level of technology has determined an efficient improvement of the treatment plans, even in dose-escalated or hypofractionated regimens. This is particularly true for moderate and severe rectal toxicities, which had an incidence close to $20 \%$ in the 1990 s while in the most recent publications they have fallen below $7 \%-10 \%$.

However, an analogous result has not yet been reached in urinary symptoms. This is mainly due to the difficulty in sparing the bladder, which is partially but unavoidably included in the target volume, as well as to the clear lack of knowledge concerning the predictors of urinary toxicity. The main reason for this deficiency is probably the difficulty in following for a sufficiently long time a large number of patients whose clinical and dosimetry data need to be individually and prospectively collected with proper evaluation of urinary symptoms. Some important symptoms (e.g., incontinence) may indeed continuously arise after radiation therapy and their incidence reaches a plateau only many years (typically $5-8$ ) after treatment. The fulfilment of reliable models of radiation-induced urinary sequelae is therefore made particularly challenging by the complexity of symptoms, their evolution over time, and the strong dependence on the baseline situation.

It is widely acknowledged that radiation-induced toxicity is a multifactorial problem, depending not only on the delivered 
dose and involving many complex biological processes in the irradiated tissues responding to cellular injury. Individual biological background and expression patterns, premorbid conditions as well as the cell microenvironment are important factors in the development of side effects, although their contributions and interaction are still mostly unknown. The ability to predict which patients are more likely to experience urinary toxicity may improve the potential of individualizing treatment with respect to several aspects concerning the choice of the therapeutic strategy, dose prescription, fractionation, and use of supportive therapies.

Only in recent years has the radiation-induced urinary syndrome been the object of large-scale prospective observational trials specifically devoted to investigating the association of patient and treatment features with acute/late urinary toxicity. The preliminary findings allowed a first attempt at predictive modeling which can serve as a basis for optimization of patient selection and treatment planning.

In this narrative review, we report on the current knowledge of the radiation-induced urinary syndrome.

\section{The radiation-induced urinary syndrome - facts and figures}

The term "urinary toxicity" comprises a wide variety of symptoms - including urinary frequency, obstruction and stricture, hematuria, dysuria or incontinence - with very different time patterns and different impacts on the individual patient's quality of life (1). Yet, the degree of their effect varies highly among patients and remains uncertain unless patient-reporting tools are used in combination with physician assessments.

The response of the urinary bladder to radiotherapy can be classified into acute/subacute reactions, occurring during radiotherapy and within 3-6 months after treatment completion, and late reactions, which start to appear 6 months after therapy and often occur many years later. The pathophysiology of urinary radiation injury is still not completely understood. The mechanisms of radiation damage affect the urothelium, the vasculature and the detrusor muscles (1). After irradiation, the urothelium exhibits nuclear irregularity and cellular edema, with disruption of the polysaccharide layer; this causes contact between hypertonic urine and isotonic tissue, resulting in tissue inflammation and early urinary symptoms (2). Vascular ischemia, edema and cellular demolition cause depletion of bladder smooth muscle and proliferation of fibroblasts, with consequent decreased bladder compliance and capacity up to hemorrhagic cystitis. Fibrosis leading to occlusion of the urethral lumen is an important factor for the onset of urethral strictures after radiotherapy (3), as well as being likely associated with reduced urinary functionality in terms of urgency and incontinence symptoms.

At the clinical level, urinary toxicity is usually graded using the Common Terminology Criteria for Adverse Events (CTCAE): version 4.03 is the most recent release of these scoring criteria (4). Toxicity is graded from 0 to 5 , with grading referring to the severity of the effect. Grade 0 indicates no adverse effect, grade 1 describes mild symptoms with no indication for intervention, grade 2 denotes moderate symptoms requiring minor, local or non-invasive intervention, and grade 3-4 are severe effects requiring hospitalization or urgent intervention and limiting self-care activities of daily living. Any death resulting from late complications of radiation is considered grade 5 . Grade 2 toxicities include (a) moderate urinary frequency/ urgency with an indication for medical management; (b) symptomatic hematuria requiring the positioning of a urinary catheter or bladder irrigation; (c) urethral obstruction needing dilation and/or the insertion of a urinary or suprapubic catheter; (d) incontinence requiring pads; (e) urinary retention leading to placement of a urinary/suprapubic or intermittent catheter, and (f) fistula requiring non-invasive intervention. Grade 3 effects comprise (a) gross hematuria requiring transfusion, hospitalization, hyperbaric oxygen therapy, radiological or operative intervention; (b) symptomatic urinary tract obstruction with altered organ function needing surgical intervention; (c) urinary incontinence necessitating clamps, collagen injections or surgery; (d) urinary retention with an indication for elective operative or radiological intervention; (e) fistula requiring radiological, endoscopic or surgical intervention or permanent urinary diversion.

The incidence of acute/late radiation-induced urinary toxicity in modern series pertaining to patients treated with radical radiotherapy varies widely, the variation being mainly related to prescription doses, delivery techniques, the presence and frequency of image guidance protocols, hypofractionation, and concomitant hormonal therapies. The rates of grade 1 and grade 2 symptoms in patients followed for up to 10 years are in the range of $20 \%-43 \%$ and $10 \%-46 \%$, respectively (5-16). Grade 3 urinary toxicity occurs at a rate of $2 \%-16 \%$. Obstruction, incontinence and radiation cystitis with gross macroscopic hematuria are the most commonly reported grade 3 symptoms. Table I summarizes the urinary toxicity rates as reported by the above-mentioned studies.

It has to be mentioned that the prevalence of lower urinary tract symptoms increases with age in the general population: moderate to severe symptoms are present in approximately $15 \%$ of men aged $50-59$ years and approximately $30 \%$ of men $70+$ years old. The most frequent symptom in the general population is nocturia $(17,18)$. For this reason the rates of late radiation-induced urinary toxicity could be overestimated.

The pattern of late toxicity is variable, with obstructive symptoms generally resolving with time or intervention, and urinary incontinence worsening with protracted follow-up and increasing patient age (19-21).

A last issue is related to the compelling confirmations of the consequential nature of late radiation-induced urinary toxicity, which were recently reported in large, prospectively followed cohorts $(5,9,13,22-27)$. This suggests that a relevant fraction of late urinary events are a "consequence" of the exuberant repair process following the acute inflammatory phase and it means that any effort to reduce acute toxicity may impact the occurrence of late events.

\section{Is the patient's point of view of interest? Challenges in measuring and reporting radiation-induced urinary symptoms and the importance of patient-reported outcomes}

Measuring and reporting radiation-induced urinary symptoms is a demanding task, which must take into account a complex set of symptoms evolving over time and strongly 


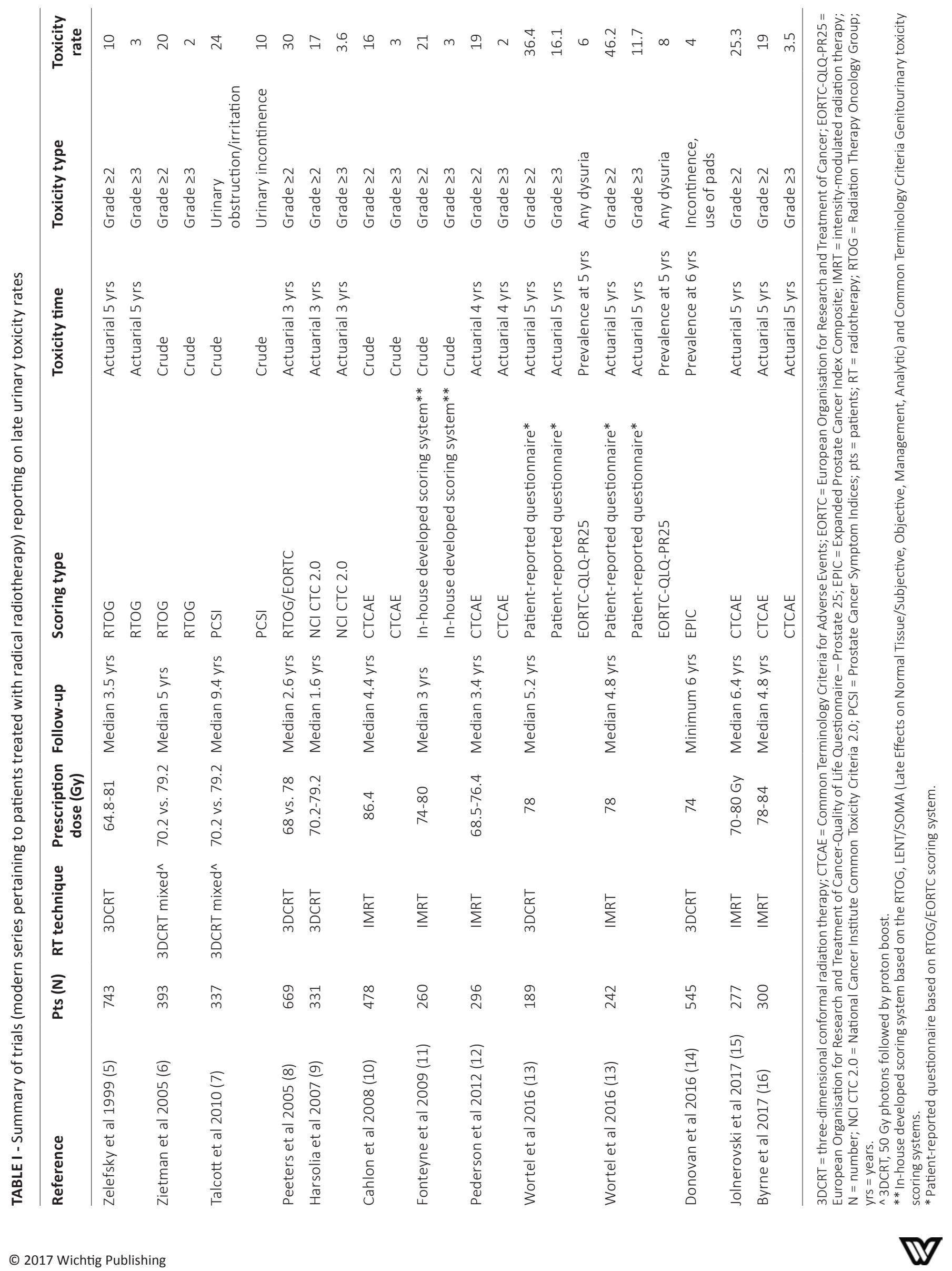


relying on individual patient characteristics and on preradiotherapy urinary functionality.

The previously described CTCAE physician-based score has been found to be much less exhaustive (and often largely different in terms of results) than patient-reported outcomes. This prompted the increasing use of specific patient-reported questionnaires, as it became evident that these instruments can describe and score many different symptoms, allowing nuances and determination of the impact of symptoms on patient-perceived quality of life.

The International Prostate Symptoms Score (IPSS (28)) thus became widely used to score obstructive symptoms and generate an overall assessment of urinary functionality (15, 29-42). Urinary incontinence can be prospectively assessed by the International Consultation on Incontinence Modular Questionnaire Short Form (ICIQ-SF (43)), which also includes the patient's perception of the impact of incontinence on quality of life $(14,44)$.

The International Consortium for Health Outcomes Measurement (ICHOM (45)) proposed the Expanded Prostate Cancer Index Composite 26-question short form (EPIC-26 (46)), which is already widely used $(14,37,39,47-50)$ and addresses all pertinent domains of prostate cancer treatment side effects including urinary obstructive symptoms, urinary incontinence and hematuria. ICHOM recognized that recommending a single instrument for comprehensive evaluation of side effects was a contentious decision, because centers of excellence already had well-established prospective programs including different patient-reported outcomes and there was no convincing indication for the superiority of one tool over another (45). However, the Consortium strongly recommended the regular use of such patient-reported instruments, from baseline up to 10-year follow-up, as part of high-quality care pathways.

\section{How can we optimize radiotherapy treatment minimizing the risk of urinary toxicity? Established dose-volume effects}

One of the most outstanding results achieved in recent years is the acknowledgment of the existence of a dosevolume effect for several urinary symptoms arising after and as a consequence of radiotherapy for prostate cancer.

Several trials reported significant associations between the dose to the urinary bladder and both acute and late urinary injury: a summary of the most relevant studies is reported in the recent review by Landoni and coworkers (51) reporting on the main findings in terms of constraints and relationships. Predominantly, the bladder seems to act as a highly serial organ (52), i.e., its functional subunits are arranged as in a chain and damage to a single subunit causes loss of functionality to the whole organ. An organ with such an architecture is highly sensitive to small volumes receiving high doses. In the particular case of the urinary bladder, reducing the volume that receives more than $75-78$ Gy or more than 8-12 Gy per week $(5,9,11,12,15,26,27,34$, 53-55) may significantly decrease the risk of acute and/or late urinary toxicity. Table II reports some details of trials highlighting the relationship between acute/late urinary toxicity and bladder doses/prescription dose (with pre- scription dose being a surrogate for dose to small bladder volumes).

An important consequence of the existence of a dosevolume effect is that any attempt to reduce the fraction of the bladder neck receiving high doses (>75-78 Gy, 2-Gyequivalent) appears to be justified. This highlights the pivotal role of image-guided radiotherapy (IGRT) in lowering the urinary toxicity risk given its potential to reduce the fraction of bladder that overlays the planning target volume, corresponding to the portion of the bladder that is irradiated at the full prescription dose. The reduction of urinary toxicity with IGRT with respect to non-IGRT reported in several studies (56-59) indirectly supports this argument.

Of note, the role of small bladder volume irradiated at high doses (or of bladder maximum dose) was also established in several trials involving post-prostatectomy settings $(24,27,60-62)$.

\section{Does the daily radiation fraction size matter? The impact of altered fractionation}

Nowadays the large majority of prostate cancer radiotherapy treatments are performed in fractionated schemes over 7-8 weeks at $2 \mathrm{~Gy} /$ fraction, delivered with intensitymodulated modalities with or without IGRT. The choice of delivering radiotherapy treatments in fractionated schedules with small fraction sizes has a sound radiobiological justification. Indeed, fractionation is a key tool for increasing the therapeutic ratio, that is, the separation between tumor control and normal-tissue damage curves which exploits the different ability of normal and tumor tissues to repair the radiationinduced damage.

However, the better sparing of normal tissues achieved with the most recent radiotherapy modalities has reawakened the interest of the radiation oncology community in hypofractionated schemes with the aim of reducing both patient discomfort and treatment costs.

Recently, various protocols including moderately to extremely hypofractionated schemes have been suggested. In particular, the 5-year efficacy results of 4 large randomized phase III trials demonstrated that hypofractionation for localized prostate cancer is non-inferior to conventional fractionation (63-66).

Nevertheless, variations to conventional schedules should be considered with caution, since extreme reduction of the number of fractions without the support of robust data on tissue radiobiological behavior might lead to unacceptably high doses to healthy tissues. This is particularly important for urinary toxicities. In the radical setting, there are some indications that hypofractionation may have a detrimental impact on urinary morbidity compared with conventional fractionation both in the acute and late stages (34, 37, 44, 66-71). On the other hand, in trials prescribing lower 2-Gy-equivalent doses, no significant impact has been reported for acute and late urinary toxicity.

How the bladder responds to variation in fraction sizes is still an open question, with evidence of a higher than previously assumed sensitivity to fractionation starting to appear both in the radical and post-prostatectomy settings (72), which has to be coupled to a steep dose-response after 75-80 Gy. 


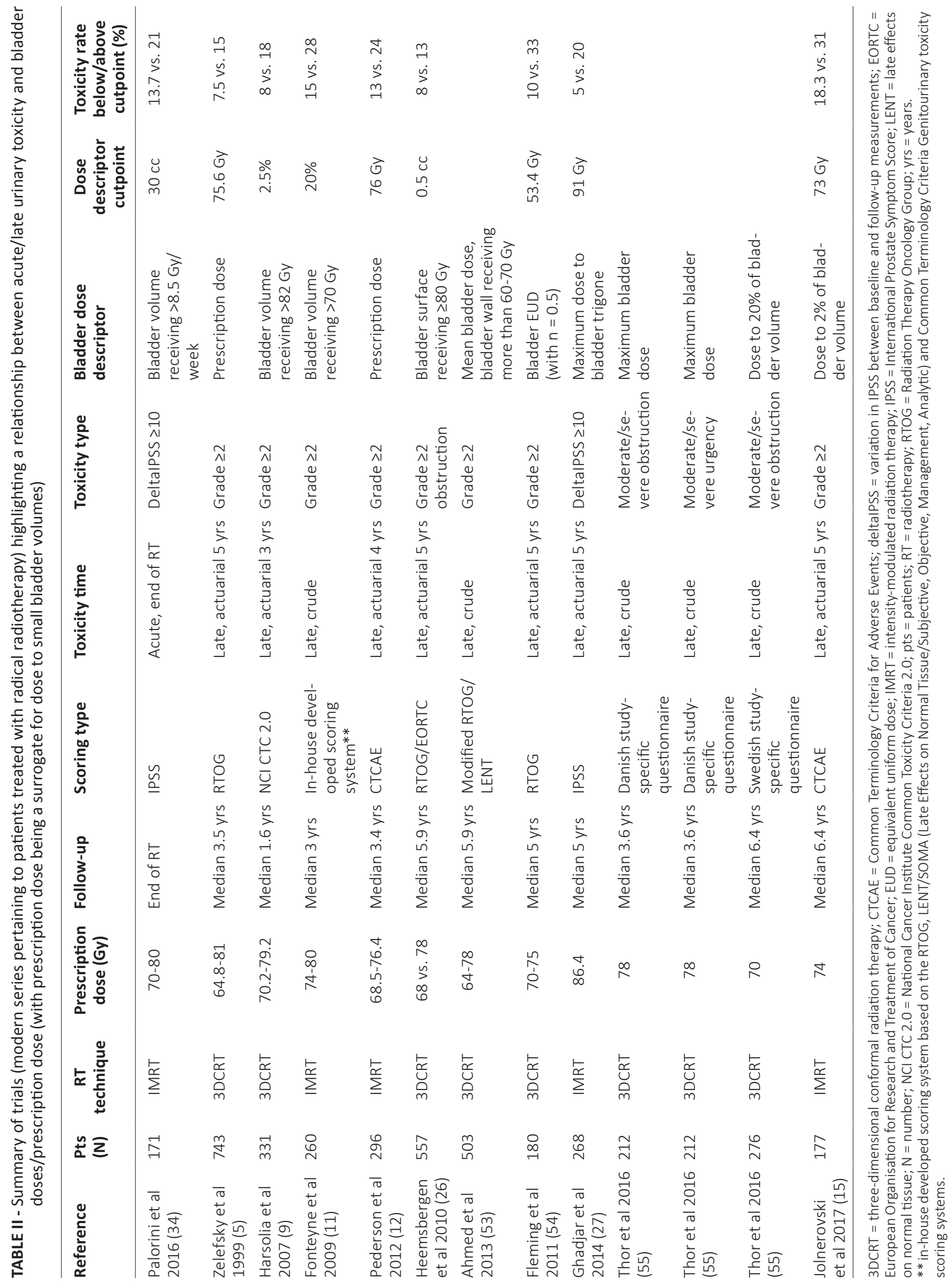




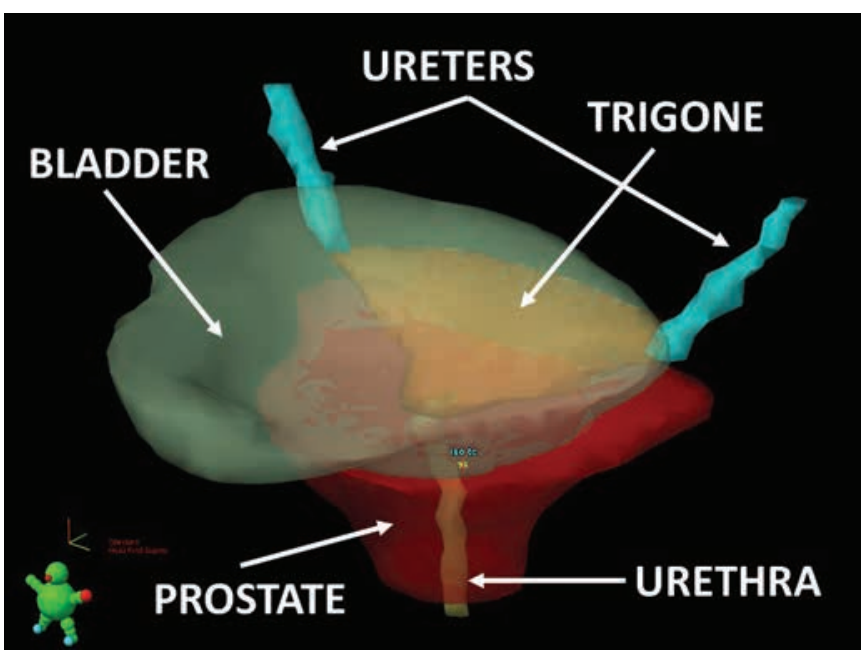

Fig. 1 - Description of bladder substructures.

These data are consistent with a very low alpha/beta ratio, a parameter describing the tissue sensitivity to fractionation. However, it must be emphasized that the confidence intervals of alpha/beta ratios that can be derived from modern practice observational clinical trials are very large, since in patients it is not possible to systematically span a wide range of fractionation schemes or prescribed doses. For this reason, the ultimate statement on bladder alpha/beta ratio will probably have to be based on new animal studies. These experiments still represent a necessary step for accurately determining the sensitivity of the bladder to fractionation.

\section{Are there exceptionally sensitive and critical substruc- tures in the bladder? Evidence of spatial effects}

Some studies from the literature underlined the need to overcome the assumption that the urinary bladder is uniformly sensitive to radiation $(26,27,31,35,36,73)$. As a matter of fact, the bladder comprises several substructures which may have distinct radiobiological behaviors and sensitivities, leading to different impacts on distinct urinary symptoms (see Fig. 1 for a summary description of these structures). The main attempt of these studies was to identify specific bladder subregions associated with urinary toxicity after radiotherapy. This could have profound consequences for treatment planning optimization to reduce the toxicity risk.

Several published results $(26,27,31,35,36,73)$ converged in the identification of the trigone dose as strongly associated with worsening of symptoms and an increased risk of severe acute and late injury. The exact mechanisms controlling the trigone are still unclear; however, as this muscle is actively involved in sphincter opening, it is realistic to claim that its damage might elicit frequency, urgency and/or incontinence symptoms. Some trials also suggested the possible presence of a threshold effect at 2-Gy-equivalent doses of 80-82 Gy $(31,35)$.

The work by Yahya et al (36) also identified the dose to the anterior-inferior portion of the bladder surface as being strongly associated with the incidence of dysuria, hematuria, and worsening of symptoms as measured by IPSS.
All these findings point to the need for refined optimization of treatment planning based on the explicit definition of critical/sensitive bladder substructures and on specific dose constraints for each bladder portion.

Of course, also the dose received by the urethra may play a role: the currently available data do not allow to distinguish the relative contribution due to bladder and urethra irradiation. A major impact of urethra irradiation (mostly associated with urethral stenosis) is expected for very high dose schedules, as reported in brachytherapy series (74).

\section{Does the individual patient matter? Clinical features as modulators of dose effects}

Many recently published studies reported relevant patient-related features that are significantly associated with an increased risk of urinary toxicity. An extensive review of these studies can be found in references 51,75 and 76 . These clinical features act as individual dose-response modifying factors, making some patients more sensitive or resistant to radiation.

A first essential risk factor for urinary toxicity, consistently described by different trials, is the baseline urinary functionality $(8,13,15,44,77,78)$, with patients having an already impaired functionality being at higher risk of experiencing severe acute and late urinary toxicity. For this reason, an evaluation of the baseline situation should be mandatory before treatment planning, also considering the possibility of stricter dose limits for some patients.

Other patient-related characteristics have been emphasized as being associated with an increased risk of worsening of acute and late symptoms: previous transurethral resection of the prostate $(8,11,16,26,44,60)$ as well as smoking $(30,79-82)$, age $(13,30,34,44,53,62,78)$, vascular comorbidities and use of cardiovascular drugs $(34,62,78)$, diabetes $(15,62,78,79)$, and use of antihypertensive medication $(30,34)$. These last patient-related characteristics are indirect markers of possible damage to the microcirculation leading to impairment of tissue oxygenation, a key step in the repair of radiation-induced tissue damage. Table III presents details on patient-related features which were found to be associated with an increased risk of acute or late urinary toxicity.

All these patient-related factors should be combined to determine the dose to critical bladder structures when developing predictive models for urinary toxicity, in order to obtain tools which have the power to individualize treatment planning and optimization. Some examples are given in references 30,34 and 62, and on the website of the Maastro Clinic, http:// www. predictcancer.org/Main.php?page=UreProstateModel.

\section{Coming to conclusions: what can we expect from the near future?}

Although much remains to be understood and investigated, our knowledge of radiation-induced urinary toxicity, and of the main dosimetry and clinical factors involved in its appearance and persistence, has increased dramatically in recent years. The key success factor leading to these first steps can surely be found in the increased awareness of the 
TABLE III - Summary of trials (modern series pertaining to patients treated with radical radiotherapy) highlighting a relationship between acute/late urinary toxicity and patient-related features

\begin{tabular}{|c|c|c|c|}
\hline Patient-related features & Reference & Odds ratio/hazard ratio & Acute/late toxicity \\
\hline & Heemsbergen et al 2010 (26) & 2.7 & Late \\
\hline & Yahya et al 2015 (78) & $2.1-3.6^{\wedge}$ & Late \\
\hline & Wortel et al 2016 (13) & 2.4 & Late \\
\hline \multirow[t]{4}{*}{ TURP } & Peeters et al 2005 (8) & 1.7 & Late \\
\hline & Fonteyne et al 2009 (11) & 1.4 & Late \\
\hline & Heemsbergen et al 2010 (26) & 3.6 & Late \\
\hline & De Langhe et al 2014 (60) & 1.4 & Late \\
\hline & Stankovic et al 2016 (79) & 17.3 & Acute \\
\hline & Solanki et al 2013 (82) & $1.5-3^{\wedge}$ & Late \\
\hline & Steinberger et al 2015 (81) & 1.8 & Late \\
\hline & Bagalà et al 2016 (80) & 14.0 & Late \\
\hline \multirow[t]{6}{*}{ Age } & Cozzarini et al 2015 (30) & $0.94 *$ & Acute \\
\hline & Palorini et al 2016 (34) & $0.94-0.96 *$ & Acute \\
\hline & Ahmed et al 2013 (53) & $1.45^{* *}$ & Late \\
\hline & Mathieu et al 2014 (62) & $1.06^{*}$ & Late \\
\hline & Yahya et al 2015 (78) & $0.91-0.96^{\wedge}$ & Late \\
\hline & Wortel et al 2016 (13) & $1.62^{* * *}$ & Late \\
\hline & Yahya et al 2015 (78) & 6.0 & Late \\
\hline & Stankovic et al 2016 (79) & 3.0 & Late \\
\hline & Jolnerovski et al 2017 (15) & 2.0 & Late \\
\hline \multirow[t]{2}{*}{ Use of antihypertensives } & Cozzarini et al 2015 (30) & 1.8 & Acute \\
\hline & Palorini et al 2016 (34) & 1.6 & Acute \\
\hline
\end{tabular}

TURP = transurethral resection of the prostate.

$\wedge$ Depending on urinary symptom.

* Continuous variable in years.

** Dichotomized at 70 years.

*** Dichotomized at 68 years.

relevance of the problem, which affects a large fraction of the older population of high-income countries, where prostate cancer is endemic and where most patients diagnosed with prostate cancer receive radiotherapy treatment.

The continuous effort towards improving our ability to predict the risk of urinary toxicity should translate into refinement of our therapeutic approaches, aiming to reduce urinary toxicity while preserving the high rates of cure for these patients. The increasing availability of information from huge databases, also coming from large international collaborations and including standardized patient-reported outcome measurements, will further boost this relevant field of research in the coming years. This will allow continuing reduction of urinary side effects and the consequent improvement 
of quality of life and reduced risk of patient regret about the choice of radiotherapy (83) in long-surviving prostate cancer patients.

\section{Disclosures}

Financial support: Tiziana Rancati is supported by Fondazione Italo Monzino. The Associazione Italiana per la Ricerca sul Cancro (AIRC) is acknowledged (grants IG14603, IG13090 and IG16087). Conflict of interest: The authors have nothing to disclose.

\section{References}

1. Rapariz-González M, Castro-Díaz D, Mejía-Rendón D; EURCIS. Evaluation of the impact of the urinary symptoms on quality of life of patients with painful bladder syndrome/chronic pelvic pain and radiation cystitis: EURCIS study. Actas Urol Esp. 2014;38(4):224-231.

2. Marks LB, Carroll PR, Dugan TC, Anscher MS. The response of the urinary bladder, urethra, and ureter to radiation and chemotherapy. Int J Radiat Oncol Biol Phys. 1995;31(5):1257-1280.

3. Mundy AR, Andrich DE. Posterior urethral complications of the treatment of prostate cancer. BJU Int. 2012;110(3):304-325.

4. CTCAE v4.03. http://evs.nci.nih.gov/ftp1/CTCAE/About.html.

5. Zelefsky MJ, Cowen D, Fuks Z, et al. Long term tolerance of high dose three-dimensional conformal radiotherapy in patients with localized prostate carcinoma. Cancer. 1999;85(11): 2460-2468.

6. Zietman AL, DeSilvio ML, Slater JD, et al. Comparison of conventional-dose vs high-dose conformal radiation therapy in clinically localized adenocarcinoma of the prostate: a randomized controlled trial. JAMA. 2005;294(10):1233-1239.

7. Talcott JA, Rossi C, Shipley WU, et al. Patient-reported longterm outcomes after conventional and high-dose combined proton and photon radiation for early prostate cancer. JAMA. 2010;303(11):1046-1053.

8. Peeters ST, Heemsbergen WD, van Putten WL, et al. Acute and late complications after radiotherapy for prostate cancer: results of a multicenter randomized trial comparing 68 Gy to 78 Gy. Int J Radiat Oncol Biol Phys. 2005;61(4):1019-1034.

9. Harsolia A, Vargas C, Yan D, et al. Predictors for chronic urinary toxicity after the treatment of prostate cancer with adaptive three-dimensional conformal radiotherapy: dose-volume analysis of a phase II dose-escalation study. Int J Radiat Oncol Biol Phys. 2007;69(4):1100-1109.

10. Cahlon O, Zelefsky MJ, Shippy A, et al. Ultra-high dose $(86.4$ Gy) IMRT for localized prostate cancer: toxicity and biochemical outcomes. Int J Radiat Oncol Biol Phys. 2008;71(2):330-337.

11. Fonteyne V, Villeirs G, Lumen N, De Meerleer G. Urinary toxicity after high dose intensity modulated radiotherapy as primary therapy for prostate cancer. Radiother Oncol. 2009;92(1):42-47.

12. Pederson AW, Fricano J, Correa D, Pelizzari CA, Liauw SL. Late toxicity after intensity-modulated radiation therapy for localized prostate cancer: an exploration of dose-volume histogram parameters to limit genitourinary and gastrointestinal toxicity. Int J Radiat Oncol Biol Phys. 2012;82(1):235-241.

13. Wortel RC, Incrocci L, Pos FJ, et al. Late side effects after image guided intensity modulated radiation therapy compared to 3D-conformal radiation therapy for prostate cancer: results from 2 prospective cohorts. Int J Radiat Oncol Biol Phys. 2016; 95(2):680-689.

14. Donovan JL, Hamdy FC, Lane JA, et al; ProtecT Study Group*. Patient-reported outcomes after monitoring, surgery, or radiotherapy for prostate cancer. N Engl J Med. 2016;375(15): 1425-1437.
15. Jolnerovski M, Salleron J, Beckendorf V, et al. Intensity-modulated radiation therapy from $70 \mathrm{~Gy}$ to $80 \mathrm{~Gy}$ in prostate cancer: six-year outcomes and predictors of late toxicity. Radiat Oncol. 2017;12(1):99.

16. Byrne K, Hruby G, Kneebone A, et al. Late genitourinary toxicity outcomes in 300 prostate cancer patients treated with doseescalated image-guided intensity-modulated radiotherapy. Clin Oncol (R Coll Radiol). 2017;29(9):617-625.

17. Rohrmann S, Katzke V, Kaaks R. Prevalence and progression of lower urinary tract symptoms in an aging population. Urology. 2016;95:158-163.

18. Malmsten UG, Molander U, Peeker R, Irwin DE, Milsom I. Urinary incontinence, overactive bladder, and other lower urinary tract symptoms: a longitudinal population-based survey in men aged 45-103 years. Eur Urol. 2010;58(1):149-156.

19. Fransson P. Patient-reported lower urinary tract symptoms, urinary incontinence, and quality of life after external beam radiotherapy for localized prostate cancer--15 years follow-up. A comparison with age-matched controls. Acta Oncol. 2008;47(5): 852-861.

20. Olsson CE, Pettersson N, Alsadius D, et al. Patient-reported genitourinary toxicity for long-term prostate cancer survivors treated with radiation therapy. Br J Cancer. 2013;108(10): 1964-1970.

21. Potosky AL, Davis WW, Hoffman RM, et al. Five-year outcomes after prostatectomy or radiotherapy for prostate cancer: the prostate cancer outcomes study. J Natl Cancer Inst. 2004;96(18): 1358-1367.

22. Zelefsky MJ, Levin EJ, Hunt M, et al. Incidence of late rectal and urinary toxicities after three-dimensional conformal radiotherapy and intensity-modulated radiotherapy for localized prostate cancer. Int J Radiat Oncol Biol Phys. 2008;70(4): 1124-1129.

23. Pinkawa $\mathrm{M}$, Holy $\mathrm{R}$, Piroth $\mathrm{MD}$, et al. Consequential late effects after radiotherapy for prostate cancer - a prospective longitudinal quality of life study. Radiat Oncol. 2010;5(1):27-35.

24. Cozzarini C, Fiorino C, Da Pozzo LF, et al. Clinical factors predicting late severe urinary toxicity after postoperative radiotherapy for prostate carcinoma: a single-institute analysis of 742 patients. Int J Radiat Oncol Biol Phys. 2012;82(1): 191-199.

25. Jereczek-Fossa BA, Zerini D, Fodor C, et al. Correlation between acute and late toxicity in 973 prostate cancer patients treated with three-dimensional conformal external beam radiotherapy. Int J Radiat Oncol Biol Phys. 2010;78(1):26-34.

26. Heemsbergen WD, Al-Mamgani A, Witte MG, van Herk $M$, Pos FJ, Lebesque JV. Urinary obstruction in prostate cancer patients from the Dutch trial ( 68 Gy vs. $78 \mathrm{~Gy}$ ): relationships with local dose, acute effects, and baseline characteristics. Int J Radiat Oncol Biol Phys. 2010;78(1):19-25.

27. Ghadjar P, Zelefsky MJ, Spratt DE, et al. Impact of dose to the bladder trigone on long-term urinary function after high-dose intensity modulated radiation therapy for localized prostate cancer. Int J Radiat Oncol Biol Phys. 2014;88(2):339-344.

28. Barry MJ, Fowler FJ Jr, O'Leary MP, et al; The Measurement Committee of the American Urological Association. The American Urological Association symptom index for benign prostatic hyperplasia. J Urol. 1992;148(5):1549-1564.

29. Carillo V, Cozzarini C, Rancati T, et al. Relationships between bladder dose-volume/surface histograms and acute urinary toxicity after radiotherapy for prostate cancer. Radiother Oncol. 2014;111(1):100-105.

30. Cozzarini C, Rancati T, Carillo V, et al. Multi-variable models predicting specific patient-reported acute urinary symptoms after radiotherapy for prostate cancer: results of a cohort study. Radiother Oncol. 2015;116(2):185-191. 
31. Palorini F, Cozzarini C, Gianolini S, et al. First application of a pixel-wise analysis on bladder dose-surface maps in prostate cancer radiotherapy. Radiother Oncol. 2016;119(1):123-128.

32. Malik R, Jani AB, Liauw SL. External beam radiotherapy for prostate cancer: urinary outcomes for men with high International Prostate Symptom Scores (IPSS). Int J Radiat Oncol Biol Phys. 2011;80(4):1080-1086.

33. Ghadjar P, Jackson A, Spratt DE, et al. Patterns and predictors of amelioration of genitourinary toxicity after high-dose intensity-modulated radiation therapy for localized prostate cancer: implications for defining postradiotherapy urinary toxicity. Eur Urol. 2013;64(6):931-938.

34. Palorini F, Rancati T, Cozzarini C, et al. Multivariable models of large International Prostate Symptom Score worsening at the end of therapy in prostate cancer radiotherapy. Radiother Oncol. 2016;118(1):92-98.

35. Improta I, Palorini F, Cozzarini C, et al. Bladder spatial-dose descriptors correlate with acute urinary toxicity after radiation therapy for prostate cancer. Phys Med. 2016;32(12):1681-1689.

36. Yahya N, Ebert MA, House MJ, et al. Modeling urinary dysfunction after external beam radiation therapy of the prostate using bladder dose-surface maps: evidence of spatially variable response of the bladder surface. Int J Radiat Oncol Biol Phys. 2017;97(2):420-426.

37. Shaikh T, Li T, Handorf EA, et al. Long-term patient-reported outcomes from a phase 3 randomized prospective trial of conventional versus hypofractionated radiation therapy for localized prostate cancer. Int J Radiat Oncol Biol Phys. 2017;97(4): 722-731.

38. Chin S, Hayden AJ, Gebski V, Cross S, Turner SL. Long term patient reported urinary function following external beam radiotherapy for prostate cancer. Clin Oncol (R Coll Radiol). 2017;29(7):421-428.

39. Shahid N, Loblaw A, Chung HT, et al. Long-term toxicity and health-related quality of life after single-fraction high dose rate brachytherapy boost and hypofractionated external beam radiotherapy for intermediate-risk prostate cancer. Clin Oncol (R Coll Radiol). 2017;29(7):412-420.

40. Eriguchi $\mathrm{T}$, Yorozu A, Kuroiwa $\mathrm{N}$, et al. Predictive factors for urinary toxicity after iodine- 125 prostate brachytherapy with or without supplemental external beam radiotherapy. Brachytherapy. 2016;15(3):288-295.

41. Miyake M, Tanaka N, Asakawa I, et al. Changes in lower urinary tract symptoms and quality of life after salvage radiotherapy for biochemical recurrence of prostate cancer. Radiother Oncol. 2015;115(3):321-326.

42. Seymour ZA, Chang AJ, Zhang L, et al. Dose-volume analysis and the temporal nature of toxicity with stereotactic body radiation therapy for prostate cancer. Pract Radiat Oncol. 2015;5(5):e465-e472.

43. Avery K, Donovan J, Peters TJ, Shaw C, Gotoh M, Abrams P. ICIQ: a brief and robust measure for evaluating the symptoms and impact of urinary incontinence. Neurourol Urodyn. 2004;23(4):322-330.

44. Cozzarini C, Rancati T, Palorini F, et al. Patient-reported urinary incontinence after radiotherapy for prostate cancer: quantifying the dose-effect. Radiother Oncol. 2017 (in press). doi:10.1016/j.radonc.2017.07.029

45. Martin NE, Massey L, Stowell C, et al. Defining a standard set of patient-centered outcomes for men with localized prostate cancer. Eur Urol. 2015;67(3):460-467.

46. Wei JT, Dunn RL, Litwin MS, Sandler HM, Sanda MG. Development and validation of the expanded prostate cancer index composite (EPIC) for comprehensive assessment of healthrelated quality of life in men with prostate cancer. Urology. 2000;56(6):899-905.
47. Barocas DA, Alvarez J, Resnick MJ, et al. Association between radiation therapy, surgery, or observation for localized prostate cancer and patient-reported outcomes after 3 years. JAMA. 2017;317(11):1126-1140.

48. Venderbos LDF, Aluwini S, Roobol MJ, et al. Long-term followup after active surveillance or curative treatment: quality-oflife outcomes of men with low-risk prostate cancer. Qual Life Res. 2017;26(6):1635-1645.

49. Qi XS, Wang JP, Gomez CL, et al. Plan quality and dosimetric association of patient-reported rectal and urinary toxicities for prostate stereotactic body radiotherapy. Radiother Oncol. 2016;121(1):113-117.

50. Lane A, Metcalfe C, Young GJ, et al; ProtecT Study group. Patient-reported outcomes in the ProtecT randomized trial of clinically localized prostate cancer treatments: study design, and baseline urinary, bowel and sexual function and quality of life. BJU Int. 2016;118(6):869-879.

51. Landoni V, Fiorino C, Cozzarini C, Sanguineti G, Valdagni R, Rancati T. Predicting toxicity in radiotherapy for prostate cancer. Phys Med. 2016;32(3):521-532.

52. Valdagni $R$, Rancati T. Reducing rectal injury during external beam radiotherapy for prostate cancer. Nat Rev Urol. 2013; 10(6):345-357.

53. Ahmed AA, Egleston B, Alcantara $P$, et al. A novel method for predicting late genitourinary toxicity after prostate radiation therapy and the need for age-based risk-adapted dose constraints. Int J Radiat Oncol Biol Phys. 2013;86(4):709-715.

54. Fleming C, Kelly C, Thirion P, Fitzpatrick K, Armstrong J. A method for the prediction of late organ-at-risk toxicity after radiotherapy of the prostate using equivalent uniform dose. Int J Radiat Oncol Biol Phys. 2011;80(2):608-613.

55. Thor $\mathrm{M}$, Olsson $\mathrm{C}, \mathrm{Oh} \mathrm{JH}$, et al. Urinary bladder dose-response relationships for patient-reported genitourinary morbidity domains following prostate cancer radiotherapy. Radiother Oncol. 2016;119(1):117-122.

56. Kok D, Gill S, Bressel M, et al. Late toxicity and biochemical control in 554 prostate cancer patients treated with and without dose escalated image guided radiotherapy. Radiother Oncol. 2013;107(2):140-146.

57. Zelefsky MJ, Kollmeier M, Cox B, et al. Improved clinical outcomes with high-dose image guided radiotherapy compared with non-IGRT for the treatment of clinically localized prostate cancer. Int J Radiat Oncol Biol Phys. 2012;84(1): 125-129.

58. Gill S, Thomas J, Fox C, et al. Acute toxicity in prostate cancer patients treated with and without image-guided radiotherapy. Radiat Oncol. 2011;6(1):145.

59. Jereczek-Fossa BA, Zerini D, Fodor C, et al. Acute toxicity of image-guided hypofractionated radiotherapy for prostate cancer: nonrandomized comparison with conventional fractionation. Urol Oncol. 2011;29(5):523-532.

60. De Langhe S, De Meerleer G, De Ruyck K, et al. Integrated models for the prediction of late genitourinary complaints after high-dose intensity modulated radiotherapy for prostate cancer: making informed decisions. Radiother Oncol. 2014;112(1): 95-99.

61. Cozzarini C, Fiorino C, Deantoni C, et al. Higher-than-expected severe (grade 3-4) late urinary toxicity after postprostatectomy hypofractionated radiotherapy: a single-institution analysis of 1176 patients. Eur Urol. 2014;66(6):1024-1030.

62. Mathieu R, Arango JDO, Beckendorf V, et al. Nomograms to predict late urinary toxicity after prostate cancer radiotherapy. World J Urol. 2014;32(3):743-751.

63. Dearnaley D, Syndikus I, Mossop H, et al; CHHiP Investigators. Conventional versus hypofractionated high-dose intensity-modulated radiotherapy for prostate cancer: 
5-year outcomes of the randomised, non-inferiority, phase 3 CHHiP trial. Lancet Oncol. 2016;17(8):1047-1060.

64. Catton CN, Lukka H, Gu CS, et al. Randomized Trial of a Hypofractionated Radiation Regimen for the Treatment of Localized Prostate Cancer. J Clin Oncol. 2017;35(17):1884-1890.

65. Incrocci L, Wortel RC, Alemayehu WG, et al. Hypofractionated versus conventionally fractionated radiotherapy for patients with localised prostate cancer (HYPRO): final efficacy results from a randomised, multicentre, open-label, phase 3 trial. Lancet Oncol. 2016;17(8):1061-1069.

66. Lee WR, Dignam JJ, Amin MB, et al. Randomized phase III noninferiority study comparing two radiotherapy fractionation schedules in patients with low-risk prostate cancer. J Clin Oncol. 2016;34(20):2325-2332.

67. Pollack A, Walker G, Horwitz EM, et al. Randomized trial of hypofractionated external-beam radiotherapy for prostate cancer. J Clin Oncol. 2013;31(31):3860-3868.

68. Hoffman KE, Voong KR, Pugh TJ, et al. Risk of late toxicity in men receiving dose-escalated hypofractionated intensity modulated prostate radiation therapy: results from a randomized trial. Int J Radiat Oncol Biol Phys. 2014;88(5):1074-1084.

69. Arcangeli S, Strigari L, Gomellini S, et al. Updated results and patterns of failure in a randomized hypofractionation trial for high-risk prostate cancer. Int J Radiat Oncol Biol Phys. 2012; 84(5):1172-1178.

70. Aluwini S, Pos F, Schimmel E, et al. Hypofractionated versus conventionally fractionated radiotherapy for patients with prostate cancer (HYPRO): late toxicity results from a randomised, non-inferiority, phase 3 trial. Lancet Oncol. 2016;17(4):464-474.

71. Sanguineti G, Arcidiacono F, Landoni V, et al. Macroscopic hematuria after conventional or hypofractionated radiation therapy: results from a prospective phase 3 study. Int J Radiat Oncol Biol Phys. 2016;96(2):304-312.

72. Fiorino $C$, Cozzarini $C$, Rancati T, et al. Modelling the impact of fractionation on late urinary toxicity after postprostatectomy radiation therapy. Int J Radiat Oncol Biol Phys. 2014;90(5): 1250-1257.

73. Palorini F, Botti A, Carillo V, et al. Bladder dose-surface maps and urinary toxicity: robustness with respect to motion in assessing local dose effects. Phys Med. 2016;32(3):506-511.
74. Sullivan L, Williams SG, Tai KH, Foroudi F, Cleeve L, Duchesne GM. Urethral stricture following high dose rate brachytherapy for prostate cancer. Radiother Oncol. 2009;91(2):232-236.

75. Fiorino C, Valdagni R, Rancati T, Sanguineti G. Dose-volume effects for normal tissues in external radiotherapy: pelvis. Radiother Oncol. 2009;93(2):153-167.

76. Budäus L, Bolla M, Bossi A, et al. Functional outcomes and complications following radiation therapy for prostate cancer: a critical analysis of the literature. Eur Urol. 2012;61(1): 112-127.

77. Barnett GC, De Meerleer G, Gulliford SL, Sydes MR, Elliott RM, Dearnaley DP. The impact of clinical factors on the development of late radiation toxicity: results from the Medical Research Council RT01 trial (ISRCTN47772397). Clin Oncol (R Coll Radiol). 2011;23(9):613-624.

78. Yahya N, Ebert MA, Bulsara M, et al. Dosimetry, clinical factors and medication intake influencing urinary symptoms after prostate radiotherapy: An analysis of data from the RADAR prostate radiotherapy trial. Radiother Oncol. 2015;116(1): 112-118.

79. Stankovic V, Džamic Z, Pekmezovic T, et al. Acute and late genitourinary toxicity after 72 Gy of conventionally fractionated conformal radiotherapy for localised prostate cancer: impact of individual and clinical parameters. Clin Oncol (R Coll Radiol). 2016;28(9):577-586.

80. Bagalà $P$, Ingrosso G, Falco MD, et al. Predicting genitourinary toxicity in three-dimensional conformal radiotherapy for localized prostate cancer: a dose-volume parameters analysis of the bladder. J Cancer Res Ther. 2016;12(2):1018-1024.

81. Steinberger $\mathrm{E}$, Kollmeier $\mathrm{M}$, McBride S, Novak C, Pei X, Zelefsky MJ. Cigarette smoking during external beam radiation therapy for prostate cancer is associated with an increased risk of prostate cancer-specific mortality and treatment-related toxicity. BJU Int. 2015;116(4):596-603.

82. Solanki AA, Liauw SL. Tobacco use and external beam radiation therapy for prostate cancer: influence on biochemical control and late toxicity. Cancer. 2013;119(15):2807-2814.

83. Hoffman RM, Lo M, Clark JA, et al. Treatment decision regret among long-term survivors of localized prostate cancer: results from the Prostate Cancer Outcomes Study. J Clin Oncol. 2017;35(20):2306-2314. 